\title{
Etnocentrismo del consumidor e intención de compra en países en desarrollo
}

\section{Consumer ethnocentrism and purchasing intention in developing countries}

\begin{abstract}
Dra. Catalina González-Cabrera es profesora e investigadora de la Universidad del Azuay (Ecuador) (cgonzalez@uazuay.edu.ec) (https://orcid.org/0000-0002-1367-9337)
\end{abstract}

Lcda. Karen Trelles-Arteaga Licenciada en Estudios Internacionales por la Universidad del Azuay (Ecuador) (katrelles@es.uazuay.edu.ec) (https://orcid.org/0000-0002-4982-4073)

\section{Resumen}

Las empresas en los países con menos experiencia productiva se ven amenazadas por el ingreso de productos extranjeros, percibidos como superiores entre los consumidores, lo que pone en desventaja a los suyos. Por consiguiente, los productores locales requieren de estrategias efectivas que doten a sus géneros de ventajas competitivas frente a las importaciones. Al respecto, investigaciones sobre el comportamiento del consumidor, han identificado que las creencias, percepciones y actitudes asociadas al país de origen de los productos tienen influencia en la intención de compra. En este sentido, el presente artículo analiza el grado en el que el etnocentrismo del consumidor tiene efecto sobre la intención de compra de calzado nacional, también examina posibles factores predictores y condicionadores de la relación; para esto, se empleó una encuesta en una muestra conformada por 361 estudiantes universitarios. En ella se utilizó una versión adaptada de la escala CETSCALE para medir el nivel de etnocentrismo del consumidor más una escala que mide la intención de compra de productos nacionales. En consecuencia, se comprobó que el nivel de etnocentrismo en el consumidor influye positivamente en la intención de compra del producto nacional y que condiciona el efecto del hábito de compra sobre la intención de compra, además, que la escala CETSCALE es una herramienta que puede ser utilizada en economías en desarrollo con interesantes resultados. Las implicaciones gerenciales y limitaciones de la investigación son discutidas a detalle.

\begin{abstract}
Companies in countries with less productive experience have seen threatened by the introduction of foreign products to their local markets, perceived as superior among consumers. Therefore, local producers require effective strategies to give their products competitive advantages over imports. In this regard, research on consumer behavior has identified that the beliefs, perceptions and attitudes associated with the country of origin of the products have an influence on the purchase intention. In this way, the present article analyzes the degree to which the consumer's ethnocentrism has an effect on the intention to buy national footwear, as well as possible predictive and conditioning factors of the relationship. For such purpose, a survey was used in a sample made up of 361 university students. It was composed by an adapted version of the CETSCALE scale used to measure the level of the consumer ethnocentrism, and a scale that measures the intention to buy national and foreign products. Consequently, it was found that the level of ethnocentrism in the consumer positively influences the purchase intention of the national product and that it conditions the effect of the purchase habit on the purchase intention; in addition, it was possible to verify that the CETSCALE scale is a tool that can be used in developing economies with interesting results. The managerial implications and limitations of the research are discussed in detail.
\end{abstract}

\section{Palabras clave I keywords}

Etnocentrismo del consumidor, CETSCALE, efecto moderador, intención de compra, hábito de compra, producto nacional, producto extranjero, países en desarrollo.

Consumer Ethnocentrism, CETSCALE, Moderation effect, buying intention, buying habits, national-made products, foreign-made products, developing countries.

Cómo citar: González-Cabrera, C., y Trelles-Arteaga, K. (2021). Etnocentrismo del consumidor e intención de compra en países en desarrollo. Retos Revista de Ciencias de la Administración y Economía, 11(21), pp. 165-180. https://doi.org/10.17163/ret.n21.2021.10 


\section{Introducción}

La globalización ha facilitado el comercio de productos más allá de sus localidades, de manera que en las plazas comerciales es común encontrar productos de diversos orígenes (Karoui \& Khemakhem, 2019; Teng, 2019). En consecuencia, se ha hecho notable que ciertos productos gocen de ventajas sobre otros por su origen de fabricación; tal efecto se estudia bajo la denominación "efecto país de origen" (country of origin effect, COE) (Hamin, 2006). Este término representa la influencia positiva o negativa que el país de manufactura de un producto puede tener en los procesos de decisión de compra del consumidor o comportamientos subsecuentes (Elliott \& Cameron, 1994). Recientemente, Kausuhe et al. (2021) analizaron la influencia de la imagen de marca y el país de origen (COE) en la decisión de compra del teléfono inteligente chino Oppo en Manado (Indonesia), encontrando un efecto positivo y significativo de la imagen de marca en la lealtad, y otro efecto positivo, pero no significativo del COE en la decisión de compra. Ahora bien, lo que resulta importante en este punto, es analizar la preferencia de compra que podría existir en los consumidores hacia los productos de origen nacional en países en vías de desarrollo como Ecuador; dado que muchas personas miran la globalización como una amenaza a los intereses de sus grupos étnicos y nacionalidad, incluida la cultura del consumo (Bizumic, 2018).

Los sesgos a favor de los productos de fabricación doméstica en los consumidores se explican a través de un rasgo de personalidad llamado "etnocentrismo de consumo" (Balabanis \& Dimantopoulos, 2004), de manera que, mientras más importancia una persona le dé a la fabricación doméstica de un producto, mayor será la tendencia etnocéntrica (Huddleston et al., 2001). En efecto, la importancia del concepto radica en su capacidad de proveer a la gestión de marketing un acercamiento a las razones que subyacen la preferencia de compra de productos domésticos versus los importados, así como, por qué ciertos segmentos de consumidores prefieren bienes domésticos mientras que otros no se muestran interesados en el origen de estos (Acikdilli et al., 2017). Para Sharma et al. (1995), el etnocentrismo ha proporcionado información útil tanto para importadores como exportadores en la selección de mercados objetivo y formulación de más apropiadas estrategias de marketing; tales como, la penetración del mercado, al revisar si la conducta etnocentrista de las personas es determinante para la compra de un producto.

En Latinoamérica, Muñoz y Velandia (2014) pretendieron establecer el nivel de etnocentrismo en relación con las actitudes e intención de compra de productos de origen nacional y extranjero en los mercados de Brasil y Colombia, los hallazgos permitieron evidenciar un bajo nivel de etnocentrismo para la muestra brasilera y un nivel medio para la colombiana, sin embargo, no se identificaron diferencias actitudinales en el consumo de productos extranjeros y domésticos del mercado colombo-brasilero. No obstante, Camarena y Sandoval (2016) en un estudio realizado en Sonora, México, encontraron diferencias en los niveles de etnocentrismo entre poblaciones de una misma región y hasta la existencia de microculturas, lo que condujo a pautas diferenciadas de comportamiento alimentario. En Ecuador, Crespo y Salas (2020) a través de modelos de ecuaciones estructurales estudiaron el comportamiento del consumidor quiteño del producto "chocolate", los hallazgos demostraron que a mayor etnocentricidad del consumidor mayor era la actitud negativa hacia la compra de chocolates importados. Cabe señalar que no se ha encontrado evidencia empírica sobre efectos moderadores entre el nivel de etnocentrismo de consumo y la intención de compra de géneros locales en el Ecuador.

Por lo tanto, este estudio tiene como propósito medir el nivel de etnocentrismo de consumo en jóvenes universitarios ecuatorianos y su efecto en la intención de compra 
tanto de productos domésticos como importados, con el fin de identificar la interacción del constructo en economías en desarrollo, y también posibles factores predictores y condicionadores de la relación.

\subsection{Etnocentrismo del consumo}

El etnocentrismo fue conceptualizado por William Sumner (1906), sociólogo de la Universidad de Yale, reconocido por desarrollar los conceptos de "difusión", "endogrupo" y "etnocentrismo"; este último ha sido definido como la tendencia de la gente a rechazar a otros que son culturalmente diferentes mientras favorecen a quienes son similares. Posteriormente, el concepto fue trasladado al comportamiento del consumidor, heredando las principales premisas y propiedades del constructo original. Aplicado al consumo, el etnocentrismo ha indicado la moralidad del individuo al comprar o rehusarse a comprar productos extranjeros (Shimp, 1984; Spillan et al., 2007) y, ha situado a la importación de productos como un problema moral y social (Spillan et al., 2007). No obstante, resultados de una reciente investigación, justifican la denominación de esta actitud proteccionista como "etnocéntrica" puesto que las personas con esta característica están muy preocupadas por la fuerza de su grupo étnico y rechazan las ideas, personas y productos que pueden debilitar a su grupo de cierta manera; incluida la cultura consumista (Bizumic, 2018).

En concepto, el etnocentrismo del consumidor (en adelante CE - por sus siglas en inglés-Consumer Ethnocentrism) representa las creencias acerca de lo apropiado y moral de comprar productos de fabricación extranjera (Shimp \& Sharma, 1987). De manera que, los consumidores con un nivel alto de etnocentrismo califican de "incorrecto" a la compra de productos importados porque, desde su punto de vista, esto daña la economía doméstica y causa pérdidas de trabajos, además, consideran a los productos extranjeros como una amenaza (Sharma et al., 1995). En suma, estos consumidores son más propensos a sesgar sus juicios al estar más inclinados a acentuar los aspectos positivos de los productos domésticos y a desechar las virtudes de los productos extranjeros (Kaynak \& Kara, 2002; Shimp \& Sharma, 1987); manifestando también una actitud proteccionista hacia el bienestar económico del país y el empleo de sus compatriotas (Bizumic, 2018; Jiménez \& Gutiérrez, 2010).

\subsection{Etnocentrismo e intención de compra}

De acuerdo con Herche (1994), las percepciones de la moralidad de comprar productos importados entre miembros de un mercado, tienen mayor influencia en las decisiones e intenciones de compra que una estrategia de marketing tradicional, ya que el proceso de toma de decisión está compuesto por un componente normativo y un descriptivo (Slovic et al., 1977). Dado que el CE modela líneas de acción que se ajustan a las creencias y valores del individuo con respecto al grupo al que pertenece, este posee un carácter normativo (Shimp, 1984). En efecto, la norma subjetiva, a saber, creencias normativas y motivación para cumplir; es uno de los componentes de la teoría de la acción razonada; modelo de la psicología social que explica los elementos que influyen la intención del comportamiento de las personas; en este caso, el consumidor (Rosero \& Montalvo, 2015). Además, el CE se caracteriza por el amor y preocupación del país del individuo y la amenaza sobre los intereses económicos derivados del daño causado por las importaciones a uno mismo y al país (Sharma et al., 1995); de manera que se esperaría que la amenaza percibida de la competencia extranjera genere una reacción psicológica que provoque que los individuos se rehúsen a comprar productos importados (Jiménez \& Gutiérrez, 2010) Luque, Ibáñez, \& del Barrio, 2000; Shimp \& Sharma, 1987 y ejerzan presión sobre los demás miembros del grupo para que repliquen tal 
comportamiento (Huddleston et al., 2001). En consecuencia, se ha demostrado que el etnocentrismo de consumo está inversamente relacionado a la intención de compra de productos importados, por considerar al comportamiento como inapropiado y perjudicial para la economía doméstica (Granzin \& Painter, 2001; Jiménez \& Gutiérrez, 2010; Netemeyer et al., 1991; Shimp \& Sharma, 1987).

Por otra parte, las consecuencias del etnocentrismo sobre los productos domésticos incluyen su sobreestimación y la obligación moral de comprarlos y preferirlos (Kaynak \& Kara, 2002; Sharma et al., 1995). En efecto, varias investigaciones encontraron que cuanto mayor es el nivel de CE, más positivas son las creencias y actitudes sobre los productos fabricados localmente y mayor es la importancia de comprarlos (p. ej.: Balabanis \& Diamantopoulos, 2004; Durvasula et al., 1997; Herche, 1992; Klein, 2002; Nadiri \& Tümer, 2010; Shimp \& Sharma, 1987).

Por consiguiente, los consumidores etnocéntricos deben mostrar actitudes más positivas hacia los productos hechos en su país antes que a los productos importados, en concordancia con el sentido de obligación que tienen con este (Shoham \& Makovec, 2003). Entonces, cuando los consumidores tienen una actitud positiva hacia el producto, se espera que esa percepción sea traducida en intenciones de compra reales (Hamin, 2006; Wu et al., 2010); de manera que, mientras más etnocéntrico sea el consumidor, hay mayor posibilidad de que este elija productos de elaboración local (Nadiri \& Tümer, 2010; Shoham \& Makovec, 2003). Es así como Akbarov (2021), demostró recientemente el efecto del etnocentrismo sobre el comportamiento de compra entre diferentes categorías de productos en Azerbaiyán, un país con características específicas de consumo.

Por lo tanto, de acuerdo con lo anterior expuesto, se postula la siguiente hipótesis:

H1: El etnocentrismo del consumidor influye en la intención de compra de productos domésticos.

Por otra parte, es importante mencionar que la mayor parte de los estudios sobre el CE se han realizado en economías desarrolladas, donde generalmente los productos domésticos tienen mejor apreciación que los extranjeros (Elliott \& Cameron, 1994; Herche, 1992; Karoui \& Khemakhem, 2019). De hecho, Klein et al., (1998) indicaron que los consumidores etnocéntricos prefieren productos de origen local porque creen que su producción nacional es la mejor. Por otra parte, los resultados de la investigación de Acikdilli et al. (2017) en Turquía, apuntan a que los consumidores tienen preferencia por los productos locales si son de igual o mejor calidad que los importados, asimismo, en Vietman, Pham (2020) encontró que el comportamiento de compra del consumidor estaba significativamente influenciado por su país de origen.

Por el contrario, según Wang y Chen (2004), en un país en desarrollo un consumidor con altos niveles de etnocentrismo no necesariamente tiende a percibir a los productos domésticos como superiores a los importados, por lo que una admiración por los productos extranjeros sumada a la percepción negativa de los productos nacionales, mitigarían el efecto del CE sobre la intención de compra. Por lo tanto, es importante estudiar el efecto del CE en países en desarrollo, donde generalmente las marcas extranjeras se consideran mejores que las nacionales (Batra et al., 2000; Hamin, 2006; Kinra, 2006; Wang \& Chen, 2004). Lo anterior sugiere que el CE es capaz de explicar de manera más efectiva los sesgos positivos de los consumidores hacia los productos locales que los sesgos negativos frente a productos extranjeros (Balabanis \& Diamantopoulos, 2004; Hamin, 2006; Kausuhe et al., 2021; Klein et al., 2005; Luque et al., 2000; Supphellen \& Rittenburg, 2001). 
Con lo anterior expuesto, se plantea la siguiente hipótesis:

H2: El etnocentrismo del consumidor favorece la calidad percibida hacia $(\mathrm{H} 2 \mathrm{a})$ los productos domésticos y no tiene efecto en la calidad percibida sobre (H2b) los productos de origen extranjero.

\subsection{Factores condicionantes del efecto etnocentrista}

Ahora bien, estimando que los consumidores en países desarrollados valoran mejor a sus productos, una conducta etnocentrista guiaría la preferencia de compra de estos; por consiguiente, la calidad percibida de un producto moderaría el efecto del etnocentrismo de consumo sobre la intención de compra (Wang \& Chen, 2004). En efecto, Yagci (2001) señaló que el etnocentrismo del consumidor es un predictor de la intención de compra de productos domésticos solo cuando la competencia es originaria de un país menos desarrollado. Asimismo, Wang y Chen (2004) demostraron que, en un país en desarrollo, la relación entre el CE y la disposición a comprar productos nacionales resultó más débil para consumidores con un juicio de baja calidad de sus productos nacionales o cuando sostienen altos valores de consumo conspicuo; puesto que los consumidores en países en vías de desarrollo perciben a los productos importados como símbolos de status social.

Por otra parte, cabe señalar que los hábitos son los que guían la toma de decisiones de compra inconscientes (Ji \& Wood, 2007); y son una forma de automaticidad en la que la decisión de compra está guiada por el contexto (Wood \& Neal, 2009) y no por un análisis más minucioso de la información del producto. En este marco, en un país en desarrollo, donde las importaciones generalmente tienen significados simbólicos de marca y estatus social, el hábito de compra puede influir negativamente sobre la intención de compra. En consecuencia, es necesario conocer, en primera instancia, la influencia del hábito de compra sobre la intención de compras nacionales; y en segunda instancia, y lo que resulta novedoso, si el nivel de etnocentrismo del consumidor condiciona el efecto del hábito sobre la intención de compra. Cabe mencionar que Akbarov (2021) demostró el efecto moderador de algunas variables sociodemográficas, tales como: el género, los ingresos personales y el estado civil, pero no contrastó el rol moderador del nivel etnocentrista. Por lo tanto, se establecen las siguientes hipótesis:

H3: El hábito de compra influye negativamente en la intención de compra de productos nacionales.

H4: El etnocentrismo del consumidor condiciona el efecto del hábito de compra de calzado en la intención de compra.

\section{Materiales y método}

Esta investigación se realizó en Ecuador, país considerado en vías de desarrollo y con poca experiencia industrial. Los consumidores ecuatorianos tienden a replicar las tendencias de consumo de los países occidentales desarrollados. En efecto, la moda y el calzado son sectores altamente invadidos por marcas extranjeras que han obstaculizado el posicionamiento de las marcas ecuatorianas que, además de estar condicionadas a expandirse globalmente por los altos costos derivados de la dolarización frente a la moneda nacional del resto de competidores regionales, encuentran dentro de su entorno local un mercado saturado. En consecuencia, es necesario identificar estrategias competitivas que doten de ventajas a los productos de elaboración ecuatoriana; proponiendo al etnocentrismo del consumidor como un factor favorable al sector para 
fomentar el consumo de sus productos frente a la competencia extranjera. Para efectos de esta investigación, se eligió al calzado porque la industria tiene una amplia y especializada producción nacional; cuenta con 5800 empresas que diseñan y producen zapatos para el mercado local y extranjero (Lara, 2018). Además, el sector es de gran peso en la economía nacional; según la Cámara Nacional de Calzado (CALTU), anualmente, las ventas del sector alcanzan un monto de $\$ 600$ millones anuales, generando alrededor de 100 mil plazas de empleo directo e indirecto (Lara, 2018).

En primer lugar, el cuestionario utilizado en el estudio, recogió información sobre el sexo, edad, área de estudio y hábito de consumo. Luego, a través de la escala CETSCALE de 6-ítems, versión de Klein et al. (2005), se midió el etnocentrismo del consumidor. Originalmente, los ítems de la escala reflejan una preferencia por los productos estadounidenses sobre los fabricados en otros países, y miden la lealtad a la compra de productos estadounidenses y el rechazo a comprar, o importar, productos extranjeros (Bizumic, 2018). Entre los ítems de la escala utilizados están "solamente deberían importarse aquellos productos que no estuvieran disponibles en Ecuador" y "un verdadero ecuatoriano debería comprar siempre productos hechos en Ecuador"; la escala es medida en un formato Likert de cinco puntos de asignación donde $1=$ totalmente en desacuerdo y $5=$ totalmente de acuerdo, que se suman para obtener una calificación de entre 6 a 30 puntos en el que un mayor puntaje significa un mayor nivel de etnocentrismo. $(M=15.45, D T=4.82$; Alfa de Cronbach= .81$)$. Se creó una variable índice del etnocentrismo del consumidor $(M=2.57, D T=.80)$.

La intención de compra se midió a través de la escala de Josiassen, Assaf y Karpen (2011). Se le pidió a los encuestados indiquen su intención de compra de zapatos ecuatorianos versus importados a través de ítems como "me sentiría culpable si compro un producto importado" y "siempre que esté disponible, preferiría comprar productos nacionales", los enunciados fueron medidos con un formato Likert de cinco puntos que van de $1=$ totalmente en desacuerdo a 5=totalmente de acuerdo. Por último, se creó una variable índice de la intención de compra. $(M=2.09, D T=.82$; Alfa de Cronbach= .81). Cabe mencionar en este punto que, de acuerdo con el promedio obtenido, el índice de intención de compra de producto nacional es bajo (2.09), lo que quiere decir que los participantes del estudio prefieren comprar productos importados. La diferencia de la media con el punto medio teórico (3) resultó estadísticamente significativa $[t(360)=-20.387, p=.000]$

La calidad percibida de un producto se midió con el precio que el consumidor está dispuesto a pagar por un producto de origen extranjero y origen nacional; bajo la premisa de que cuando se carece de mayor información sobre un producto, el precio tiene efecto en la calidad percibida (Chang \& Wildt, 1994; Dodds \& Monroe, 1985; Olson \& Jacoby, 1972). A través de las preguntas ¿Cuánto está dispuesto a pagar por un par de zapatos ecuatorianos? Con intervalos de precios que van de $\$ 10$ a $\$ 70$ o más, asimismo se preguntó por los zapatos importados. Y, por último, el hábito de compra se midió con la cantidad de zapatos que se ha comprado en el año (de un par hasta nueve en adelante).

El número de cuestionarios válidos fue de 361 para el que se aplicó el método de muestreo no probabilístico por conveniencia debido a la facilidad de reclutamiento de los participantes ya que todos eran estudiantes de la Universidad del Azuay, CuencaEcuador. La muestra consistió en 213 mujeres (59\%) y 148 hombres (41\%), entre 17 a 37 años de las distintas áreas de especialización universitaria.

\section{Resultados}

La hipótesis 1 plantea que el etnocentrismo del consumidor influye en la intención de compra de productos domésticos. Para el contraste de la hipótesis se realizó 
una regresión lineal. Tal como se puede apreciar en la Tabla 1, el valor de $F=183,950$ tiene un valor $p$ menor a 0,05 por lo que se concluye que la dependencia lineal es estadísticamente significativa.

Por lo tanto, se confirma la hipótesis 1, el nivel de etnocentrismo de los participantes influye en su intención de compra de productos domésticos, además la relación señala que mientras mayor es el índice del etnocentrismo del consumidor mayor es la intención de compra del producto nacional (Véase Figura 1).

Tabla 1. Regresión lineal sobre la intención de compra de producto nacional

\begin{tabular}{|l|c|c|c|c|c|c|}
\hline & $\mathbf{R}$ & $\begin{array}{r}\mathbf{R} \\
\text { cuadrado }\end{array}$ & $\mathbf{F}$ & Beta & $\mathbf{t}$ & Sig. \\
\hline CE & 0,582 & 0,339 & 183,950 & 0,506 & 13,562 & 0,000 \\
\hline
\end{tabular}

Nota: Variable dependiente: Intención de compra.

\section{Figura 1. Dispersión, etnocentrismo del consumidor e intención de compra}

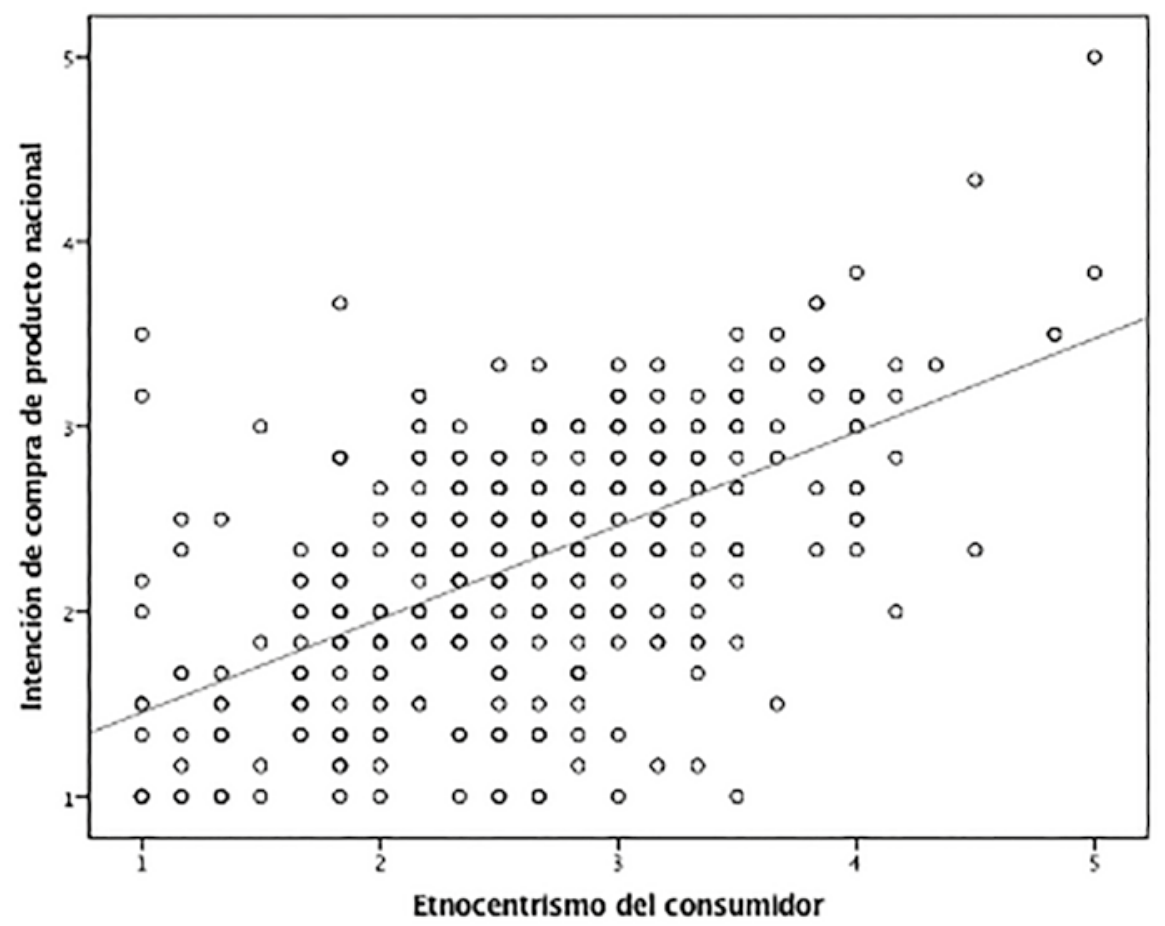

La hipótesis 2 formula "El etnocentrismo del consumidor favorece la calidad percibida hacia (a) los productos domésticos y no tiene efecto en la calidad percibida sobre (b) los productos de origen extranjero", se aplica para su contraste una regresión lineal. Se obtiene en cuanto a los productos domésticos, un valor de $F=, 682$ pero al tener un valor p mayor a 0,05 se rechaza la $\mathrm{H} 2 \mathrm{a}$. Es decir, el etnocentrismo del consumidor no influye en la percepción de la calidad de los productos domésticos. No obstante, en cuanto a los productos de origen extranjero, el valor de $F=3,352$ tiene un valor $p$ menor a 0,1 y mayor a 0,05 , lo que señala que es tendencial. Es decir, que, si se ampliase la muestra o el contexto de estudio, este dato podría cambiar. Por el momento, se rechaza la $\mathrm{H} 2 \mathrm{~b}$ al no encontrar soporte empírico suficiente para confirmar que no hay un efecto del etnocentrismo del consumidor en la calidad percibida de los productos importados (Véase Tabla 2). 
Tabla 2. Regresión lineal sobre la calidad percibida hacia el producto doméstico y extranjero

\begin{tabular}{|l|c|c|c|c|c|c|}
\cline { 2 - 7 } \multicolumn{1}{c|}{} & $\mathbf{R}$ & \begin{tabular}{c}
$\mathbf{R}$ \\
\multicolumn{1}{c|}{}
\end{tabular} & $\mathbf{F}$ & Beta & $\mathbf{t}$ & Sig. \\
\hline $\mathrm{CE}^{1}$ & 0,044 & 0,002 & 0,682 & $-0,044$ & $-0,826$ & 0,409 \\
\hline $\mathrm{CE}^{2}$ & 0,096 & 0,009 & 3,352 & $-0,096$ & $-1,831$ & 0,068 \\
\hline
\end{tabular}

1. Variable dependiente: Calidad percibida hacia el producto doméstico.

2. Variable dependiente: Calidad percibida hacia el producto extranjero.

Para la comprobación de la hipótesis 3 que plantea que el hábito de compra influye negativamente en la intención de compra de productos nacionales, bajo el argumento de que, el hábito no es una compra consciente; y que, además, en un país en desarrollo como Ecuador, la gente altamente consumidora prefiere lo importado; quienes, además, suelen tener un poder adquisitivo alto, no suelen comprar producto nacional y por tanto, presentan valores de consumo conspicuo, "lo importado es mejor".

Para el contraste de esta hipótesis se aplicó una regresión lineal simple, tal como se aprecia en la Tabla 3, de acuerdo con los valores del estadístico $F$ del ANOVA, [F= $(1,359)=11,482, p<.0001]$ se confirma la H3. Además, a través del coeficiente beta, se puede establecer que existe una relación negativa entre el hábito de compra y la intención de comprar producto nacional $[b=-0.17, t(359)=-3,388, p<.0001]$. Véase también en la Figura 2 que al aumentar el hábito de compra disminuye la intención de compra de calzado nacional.

Tabla 3. Regresión lineal sobre la intención de compra de productos nacionales

\begin{tabular}{|l|c|c|c|c|c|c|}
\cline { 2 - 7 } \multicolumn{1}{c|}{} & $\mathbf{R}$ & $\begin{array}{c}\mathbf{R} \\
\text { cuadrado }\end{array}$ & $\mathbf{F}$ & Beta & $\mathbf{t}$ & Sig. \\
\hline Hábito de compra & $-0,176$ & 0,031 & 11,482 & $-0,176$ & $-3,388$ & 0,001 \\
\hline
\end{tabular}

Variable dependiente: Intención de compra

Figura 2. Regresión lineal del hábito de compra sobre la intención de compra

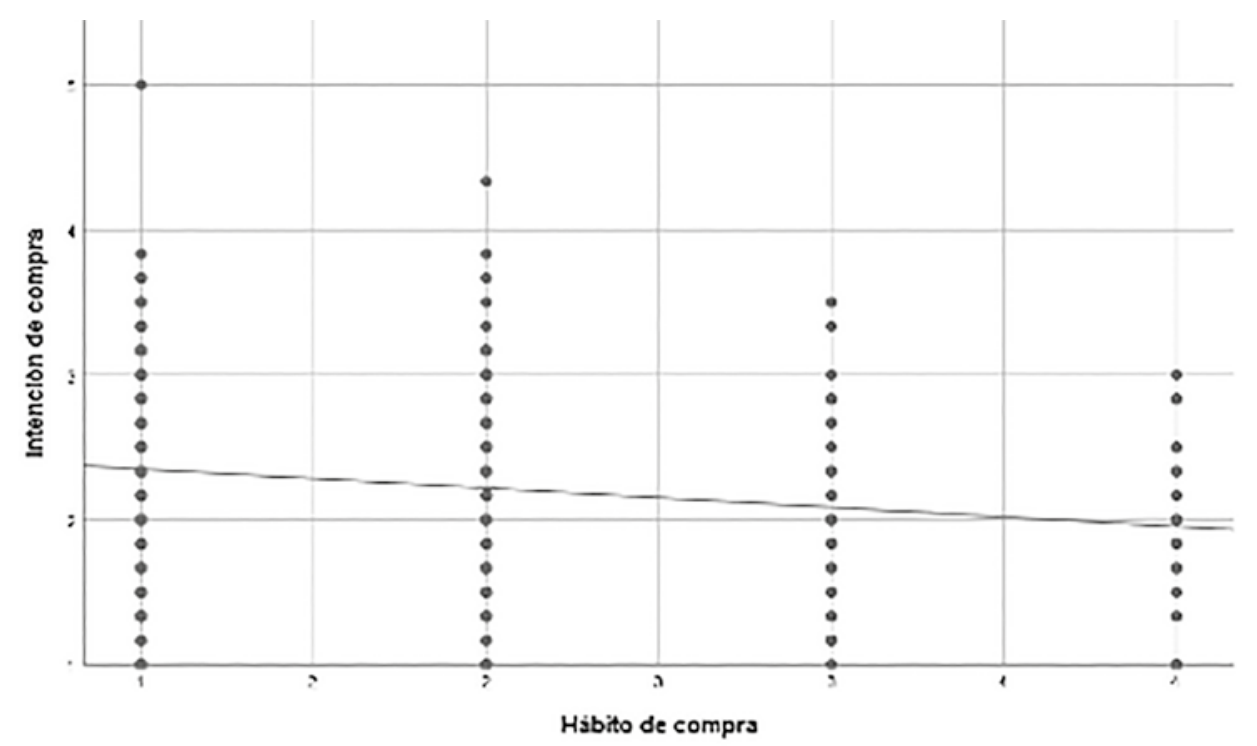


Por último, la hipótesis 4 formula que el nivel de etnocentrismo del consumidor condiciona el efecto del hábito de compra sobre la intención de comprar un producto de origen nacional. (Véase modelo en Figura 3). Para el contraste de esta hipótesis, se aplicó el modelo 1 de moderación de la macro PROCESS elaborada por Hayes (2013, 2018) que se basa en la regresión lineal múltiple.

Figura 3. Modelo condicional

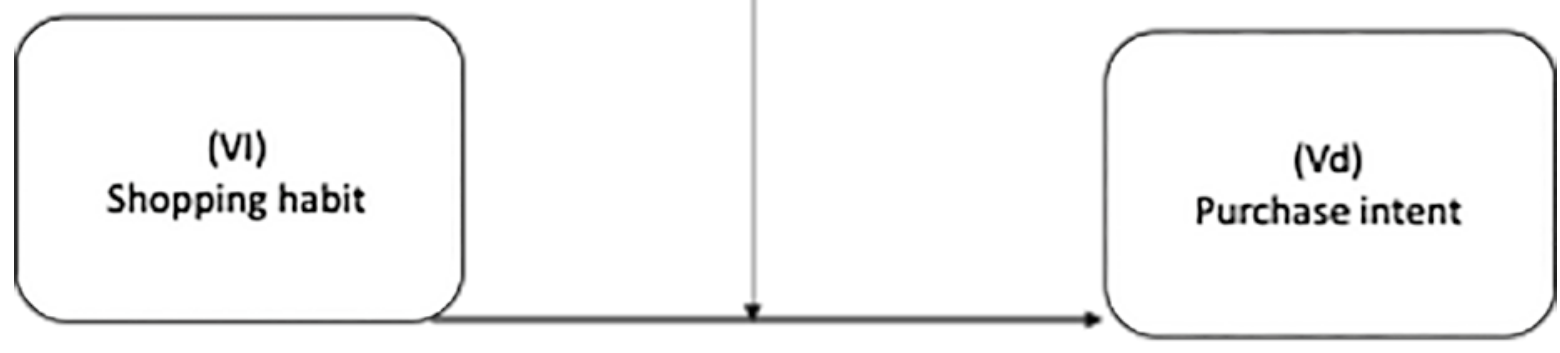

Los resultados de los análisis confirman la hipótesis 4. Por una parte, se obtiene una interacción estadísticamente tendencial entre el hábito de compra y el nivel de etnocentrismo ( $b=-.189, p=.057)$, además, se encontró un efecto moderador o condicionante del etnocentrismo del consumidor en la intención de compra (Véase Tabla 4). Es decir, cuando el hábito de compra es alto, la intención de compra de producto nacional desciende, pero esto sucede solamente en las personas con alto $(B=-.322, p=.008)$ y medio nivel de etnocentrismo $(B=-.164, p=.034)$ (Véase Figura 4). Este resultado se discute en el siguiente apartado.

\section{Tabla 4. Análisis de moderación del etnocentrismo del consumidor en la intención de compra de producto nacional}

\begin{tabular}{l|c|c|c|cc|}
\hline \multicolumn{1}{c|}{ Variable moderadora (W) } & B & ES & p & \multicolumn{2}{c|}{$\begin{array}{c}\text { IC 95\% } \\
\text { LI LS }\end{array}$} \\
\hline Etnocentrismo del consumidor (bajo) & -.038 & .094 & .679 & -.223 & .146 \\
\hline Etnocentrismo del consumidor (medio) & -.164 & .077 & .034 & -.317 & -.011 \\
\hline Etnocentrismo del consumidor (alto) & -.322 & .122 & .008 & -.562 & -.082 \\
\hline
\end{tabular}

Nota: $\mathrm{B}=$ coeficiente interacción. $\mathrm{ES}=$ error estándar. $\mathrm{IC}=$ intervalo de confianza. $\mathrm{LI}=$ Límite inferior, LS= Límite superior. 
Figura 4. Interacción de cómo el etnocentrismo interfiere entre el hábito de compra y la intención de compra de producto nacional

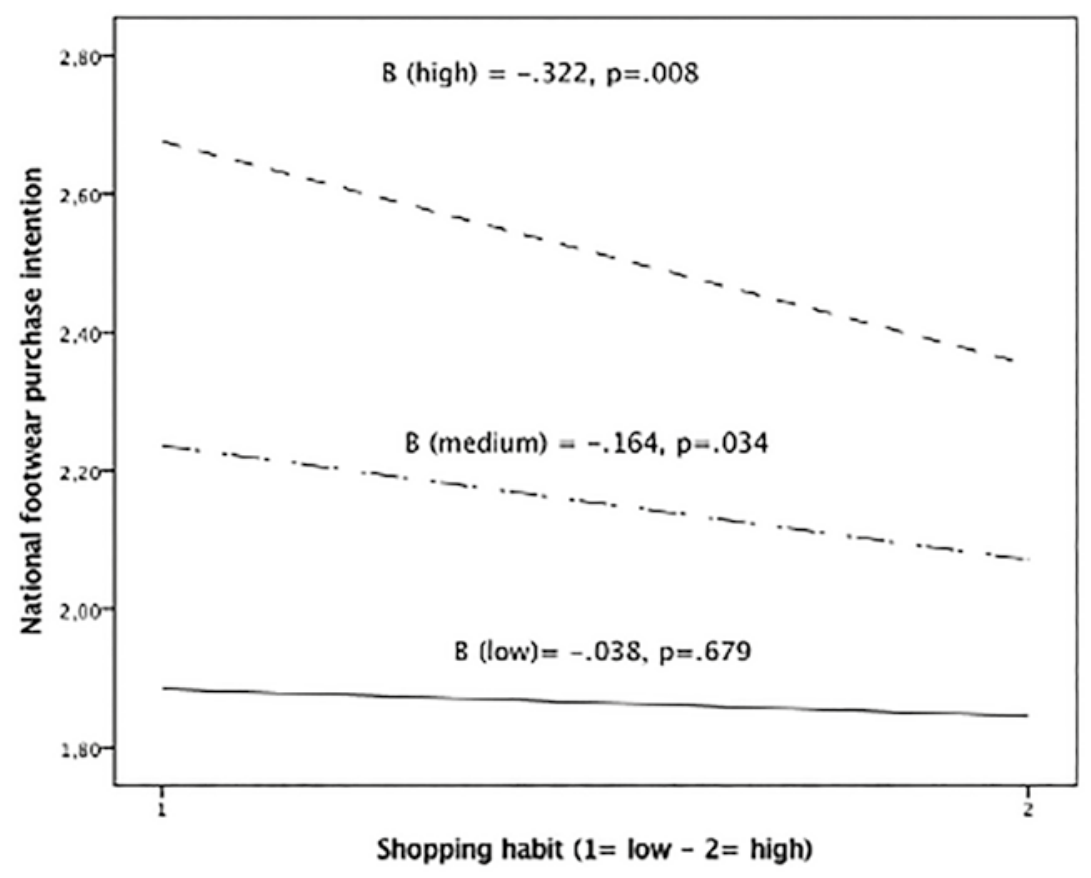

Con la técnica Johnson-Neyman (Hayes 2013, 2018) se pudo conocer que el punto exacto en el cual la interacción comienza a ser estadísticamente significativa fue a partir de 2.42 (del rango de la variable moderadora "nivel de etnocentrismo del consumidor" de 1 a 5) (Véase Figura 5). Antes de eso, no se daba un efecto condicional del etnocentrismo del consumidor en la intención de compra.

Figura 5. Región de la significancia del efecto condicional (técnica Johnson Neyman)

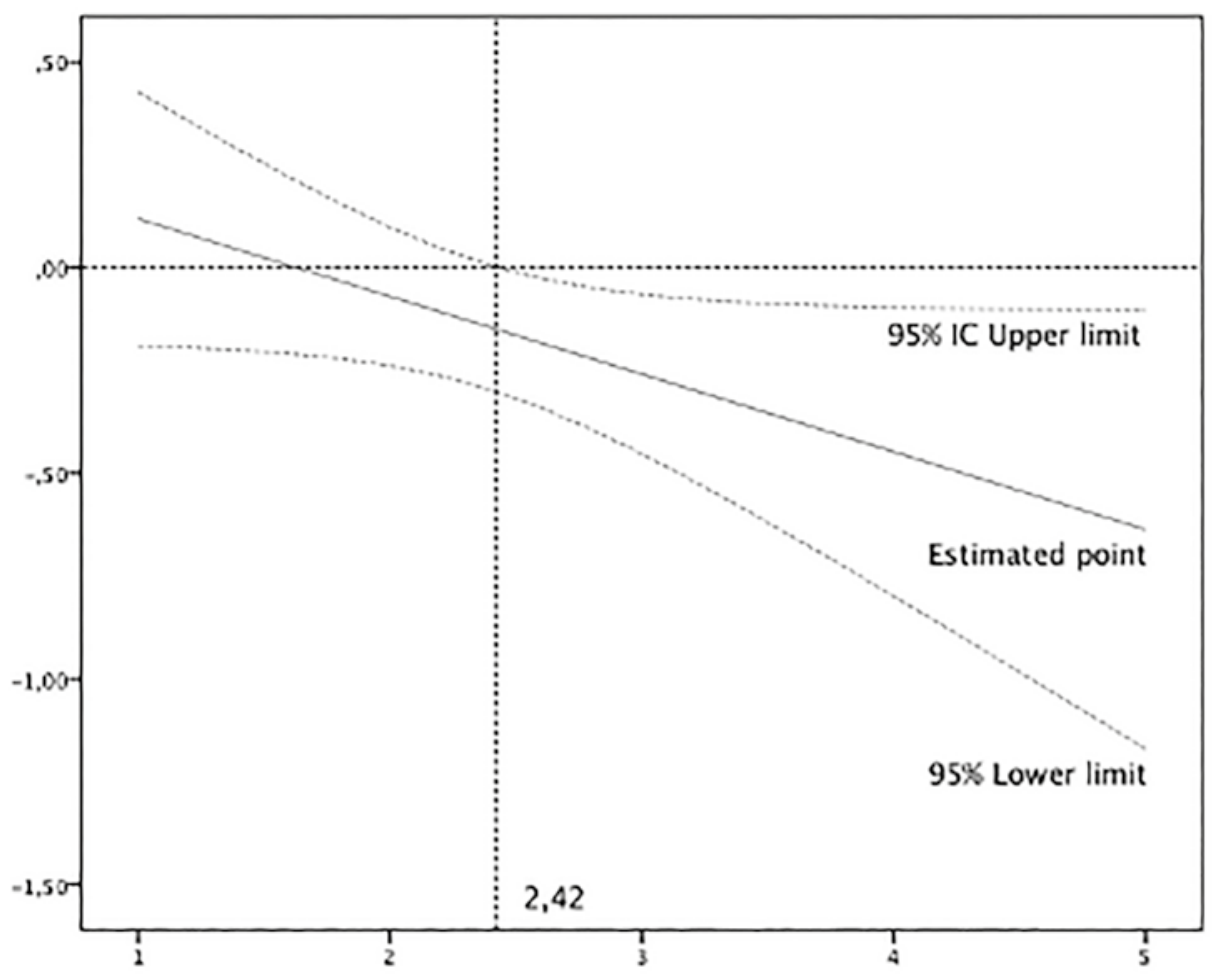




\section{Conclusiones y discusión}

En primer lugar, este estudio demuestra la validez y fiabilidad de la escala CETSCALE en el Ecuador, tal como el estudio de Crespo y Salas (2018) pero con otro tipo de producto, ellos analizaron el consumo de chocolate, en este caso, el calzado; de esta manera se aporta a la investigación del etnocentrismo del consumidor en países en vías de desarrollo. Como se mencionó en la literatura, la mayor parte de estudios sobre el tema se han realizado en países desarrollados donde generalmente los productos locales tienen mayor aceptación por su percepción de calidad derivada de la imagen del país al que pertenecen (Wang \& Chen, 2004). En efecto, a través de la escala de intención de compra, la muestra que conformó este estudio demostró que existe un nivel muy bajo de preferencia por los productos nacionales $\mathrm{y}$, asimismo, señalaron no sentirse moralmente mal si compran un producto de origen extranjero.

A partir de lo anterior, y considerando que estudios previos demostraron que existe una significativa relación entre el CE y la intención de comprar bienes producidos en el país, fue necesario investigar en qué manera y medida el CE puede influir en la decisión de compra en países donde se tiene mayor preferencia de los productos extranjeros sobre los productos nacionales. De acuerdo con Shimp y Sharma (1987), la universalidad del concepto debe tener la misma habilidad predictiva de las creencias y percepciones de los consumidores en cualquier contexto económico y, por lo tanto, debe ser capaz de predecir preferencias e intenciones de compra tal y como lo hace en economías avanzadas. En consecuencia, la $\mathrm{H} 1$ demostró que, en la muestra del estudio, el CE afecta positivamente la intención de compra del producto nacional. A pesar de que el nivel de $\mathrm{CE}$ del grupo es generalmente bajo $(\mathrm{M}=2.57)$, este demostró afectar positivamente la intención de compra de productos domésticos.

De la misma manera, según Klein et al. (2005), en entornos donde las marcas extranjeras se perciben como superiores a las locales, el efecto del CE se expresa en percepciones más positivas acerca de los productos domésticos y con poco o ningún efecto sobre las marcas extranjeras. De esta manera, se planteó la H2, con el fin de examinar si el etnocentrismo del consumidor favorecía la calidad percibida hacia (a) los productos domésticos y no tendría efecto en la calidad percibida sobre (b) los productos de origen extranjero. Sin embargo, se obtuvo que el etnocentrismo del consumidor no influye en la percepción de la calidad de los productos domésticos. No obstante, el resultado resultó marginalmente estadístico en cuanto a los productos de origen extranjero. Es decir, que si se ampliase la muestra o cambiase el contexto de estudio este dato podría ser estadísticamente significativo. En adición a lo encontrado, se puede afirmar que, el etnocentrismo en el consumo es un constructo moral e independiente de la evaluación de calidad que el consumidor tiene sobre un producto nacional. Lo anterior también se sustenta en hallazgos de la investigación de Teng (2019), quien demostró que el etnocentrismo del consumidor no tiene un efecto de moderación significativo en la relación entre la calidad percibida y la intención de compra de productos nacionales.

Ahora bien, la literatura sobre el comportamiento del consumidor nos sugiere que existen motivaciones sociales que determinan la intención de compra. En este sentido, se estableció al consumo conspicuo, medido a través de la frecuencia de compra, como determinante en el proceso de decisión de compra. En efecto, cuando las personas con frecuencia han realizado una respuesta en contextos particulares, el contexto puede llegar a desencadenar la respuesta directamente en el sentido de que no requiere objetivos e intenciones de apoyo (Wood \& Neal, 2009). De esa manera, se sostuvo que la intención de compra de productos nacionales se vería afectada cuando el consumidor realiza compras inconscientes, a partir de experiencias y no necesariamente de los atri- 
butos intangibles del producto como su lugar de fabricación. Efectivamente, los resultados de la H3 confirmaron lo expuesto y por lo tanto, los hábitos de compra afectan negativamente la intención de compra de productos nacionales.

Por otra parte, considerando que empíricamente el CE va más allá de las evaluaciones de calidad y representa un comportamiento moral (Shimp \& Sharma, 1987); se estimó que para que el CE favorezca la intención de compra de un producto nacional, es necesario que el consumidor conozca el origen de fabricación. Para verificar lo anteriormente expuesto, se evaluó al CE como un moderador del hábito de compra en la intención de compra. En consecuencia, la $\mathrm{H} 4$ confirmó la premisa y, resulta que, en el consumidor habitual la conducta etnocentrista no motiva la compra de productos nacionales. Esto se puede deber a que los consumidores habituales son personas con mayor nivel adquisitivo y se interesan más por la imagen de marca y el estatus social que esta puede representar.

En síntesis, una vez demostrada la influencia del etnocentrismo sobre la intención de compra de productos nacionales; el etnocentrismo del consumidor tiene importantes implicaciones prácticas para empresas y gobiernos. Por una parte, los productores locales se favorecen del etnocentrismo del consumidor al tener una percepción sobre la ventaja que podrán tener sobre el mercado local por el hecho de que su producción es nacional y por el conocimiento de que su participación en el mercado implica una ganancia para toda la sociedad. Esto es necesario, puesto que, en contextos económicos como el ecuatoriano, las Pymes locales intentan sobrevivir en un mercado relativamente pequeño y saturado por productos extranjeros. En ese sentido, este estudio se desarrolló con el propósito de identificar implicaciones prácticas que beneficien el fortalecimiento y expansión de la industria local; al proveer a los pequeños negocios locales, información acerca de la ventaja que sus productos tienen por el hecho de ser de fabricación nacional.

En la actualidad, es importante dirigir los planes de marketing al consumidor considerando sus factores sociales, personales, culturales y psicológicos, tal como Ortega-Vivanco (2020) concluye en su estudio realizado en la coyuntura actual causada por la COVID-19; en este demuestra que existe una relación significativa entre los cambios de los hábitos de consumo y los factores que influyen el comportamiento del consumidor en los tiempos de pandemia, tales como el incremento de las preferencias en el consumo de productos locales y la necesidad por parte de las empresas de diseñar estrategias de marketing digital enfocadas más en el cliente que en el producto, que satisfagan sus necesidades pos-COVID-19, claramente asociadas al ahorro y al impulso de los emprendimientos locales.

Paralelamente, el gobierno u organizaciones buy local pueden guiar campañas que evoquen un sentimiento patriótico; es decir, proponer la compra de productos locales como una forma de ser ecuatoriano; y a la vez representar a las importaciones como una forma de amenaza a la economía, destacando los perjuicios que estas causan a toda la sociedad. Todo esto como resultado de haber demostrado que el efecto del CE sobre la intención de compra es una conducta netamente nacionalista independientemente de las consideraciones de precio o calidad (Shankarmahesh, 2006).

Finalmente, se sugieren futuras líneas de investigación, tales como determinar el punto emocional-tradicional del consumidor y su relación con su nivel etnocentrista; así como, se pueden identificar otros factores condicionantes del CE sobre la intención de compra como el consumo conspicuo; de manera que se pueda potenciar el efecto del CE para beneficio de la industria local. Además, es importante ampliar el estudio a otras industrias con el fin de confirmar los resultados encontrados en este estudio y generalizarlos para demás sectores. Asimismo, en la revisión de la literatura 
se mencionó la corta investigación del CE en economías en desarrollo; por lo que se propone estudiar y aplicar la CETSCALE en otros espacios de similares características al Ecuador y con otras categorías de productos.

\section{Referencias}

Acikdilli, G., Ziemnowicz, C., \& Bahhouth, V. (2017). Consumer Ethnocentrism in Turkey: Ours are Better than Theirs, Journal of International Consumer Marketing, 30(1), 45-57. https://doi.org/10.1080/08961530.2017.1361882

Akbarov, S. (2021). Consumer ethnocentrism and purchasing behavior: moderating effect of demographics. Journal of Islamic Marketing,12(1). https://doi.org/10.1108/JIMA-02-2020-0047

Balabanis, G., \& Diamantopoulos, A. (2004). Domestic Country Bias, Country-of-Origin Effects, and Consumer Ethnocentrism: A Multidimensional Unfolding Approach. Journal of the Academy of Marketing Science,32(1), 80-95. https://doi.org/10.1177/0092070303257644

Batra, R., Ramaswamy, V., Alden, D., Steenkamp, J., \& Ramachander, S. (2000). Effects of Brand Local and Nonlocal Origin on Consumer Attitudes in Developing Countries. Journal of Consumer Psychology, 9(2), 83-95. https://doi.org/10.1207/S15327663JCP0902_3

Bizumic, B. (2018). Effects of the dimensions of ethnocentrism on consumer ethnocentrism: An examination of multiple mediators.International Marketing Review, 36(5), 748-770. https://doi.org/10.1108/IMR-04-2018-0147

Camarena-Gómez, D.M., \& Sandoval-Godoy, S.A. (2016). Etnocentrismo y comida típica regional: una caracterización de las preferencias y consumo de la población urbana de Sonorall Ethnocentrism and Traditional Regional Food: A Characterization of the Preferences and Consumption of the Sonora Urban Population. Razón y Palabra, 20(3_94), 501-516. https://bit.ly/39SMjNu

Crespo, G., \& Salas, R. (2018). Etnocentrismo y su influencia en el comportamiento del consumidor de chocolate en el cantón Quito. Revista Yura: Relaciones Internacionales, (14) 121-142. https://bit.ly/3c4uei3

Chang, T.Z., \& Wildt, A.R. (1994). Price, product information, and purchase intention: An empirical study. Journal of the Academy of Marketing Science: Official Publication of the Academy of Marketing Science, 22(1), 16-27. https://doi.org/10.1177/0092070394221002

Dodds, W., \& Monroe, K. (1985). The effect of brand and price information on subjective product evaluations. Advances in Consumer Research, 126(2), 129-138. https://doi.org/10.1037/a0026025

Durvasula, S., Andrews, J.C., \& Netemeyer, R.G. (1997). A Cross-Cultural Comparison of Consumer Ethnocentrism in the United States and Russia. Journal of International Consumer Marketing, 9(4), 73-93. https://doi.org/10.1300/J046v09n04_05

Elliott, G.R., \& Cameron, R.C. (1994). Consumer Perception of Product Quality and the Country-of-Origin Effect. Journal of International Marketing, 2(2), 49-62.

https://doi.org/10.1177/1069031X9400200204

Granzin, K.L., \& Painter, J.J. (2001). Motivational influences on "buy domestic" purchasing: Marketing management implications from a study of two nations. Journal of International Marketing, 9(2), 73-96. https://doi.org/10.1509/jimk.9.2.73.19883

Hamin, E.G. (2006). A less-developed country perspective of consumer ethnocentrism and "country of origin" effects: Indonesian evidence. Asia Pacific Journal of Marketing and Logistics, 18(2), 7992. https://doi.org/10.1108/13555850610658246

Hayes, A. (2013). Introduction to mediation, moderation, and conditional process analysis. The Guilford Press. https://doi.org/978-1-60918-230-4

Hayes, A.F. (2018). Introduction to Mediation, Moderation, and Conditional Process Analysis, Second Edition: A Regression-Based Approach. The Guilford Press (2.a).

Herche, J. (1992). A note on the predictive validity of the CETSCALE. Journal of the Academy of Marketing Science, 20(3), 261-264. https://doi.org/10.1007/BF02723413 
Herche, J. (1994). Ethnocentric Tendencies, Marketing Strategy and Import Purchase Behaviour. International Marketing Review, 11(3), 4-16. https://doi.org/10.1108/02651339410067012

Huddleston, P., Good, L.K., \& Stoel, L. (2001). Consumer ethnocentrism, product necessity and Polish consumers' perceptions of quality. International Journal of Retail EF Distribution Management, 29(5), 236-246. https://doi.org/10.1108/09590550110390896

Ji, M.F., \& Wood, W. (2007). Purchase and consumption habits: Not necessarily what you intend. Journal of Consumer Psychology, 17(4), 261-276. https://doi.org/10.1016/S1057-7408(07)70037-2

Jiménez, N., \& Gutiérrez, S. (2010). Socio-Psychological Antecedents of the Buying of Foreign Products: Consumer Ethnocentrism and Animosity. Revista Española de Investigación En Marketing ESIC, 14, 7-24.

Josiassen, A., Assaf, G., \& Karpen, I. (2011). Consumer ethnocentrism and willingness to buy: Analyzing the role of three demographic consumer characteristics. International Marketing Review, 28(6), 627-646. https://doi.org/10.1108/02651331111181448.

Karoui, S., \& Khemakhem, R. (2019). Consumer ethnocentrism in developing countries. European Research on Management and Business Economics, 25, 63-71. https://doi.org/10.1016/j.iedeen.2019.04.002

Kausuhe, J.N., Worang, F.G., \& Gunawan, E.M. (2021). The influence of brand image and country of origin on purchase decision of Oppo Smartphone in Manado. Journal EMBA, 9(1). https://doi.org/10.35794/emba.v9i1.32041

Kaynak, E., \& Kara, A. (2002). Consumer perceptions of foreign products. European Journal of //Marketing, 36(7-8), 928-949. https://doi.org/10.1108/03090560210430881

Kinra, N. (2006). The effect of country-of-origin on foreign brand names in the Indian market. Marketing Intelligence and Planning, 24(1), 15-30. https://doi.org/10.1108/02634500610641534

Klein, J.G., Ettenson, R., \& Morris, M.D. (1998). The animosity model of foreign product purchase: an empirical test in the People's Republic of China. The Journal of Marketing, 62(1), 89-100. https://doi.org/10.2307/1251805

Klein, J.G. (2002). Us versus them, or us versus everyone? delineating consumer aversion to foreign goods. Journal of International Business Studies, 33(2), 345-363. https://doi.org/10.1057/palgrave.jibs.8491020

Klein, J.G., Ettenson, R., \& Krishnan, B.C. (2005). Extending the construct of consumer ethnocentrism: When foreign products are preferred. International Marketing Review, 23(3), 304-321. https://doi.org/10.1108/02651330610670460

Lara, F. (2018). Zapatos ecuatorianos, con un mercado cuesta arriba. Revista Vistazo. https://bit. ly/39ZKSfU

Luque, T., Ibáñez, J., \& del Barrio, S. (2000). Consumer ethnocentrism measurement. An assessment of the reliability and validity of the CETSCALE in Spain. European Journal of Marketing, 34(11/12), 1353-1374. https://doi.org/10.1108/03090560010348498

Muñoz, C.A., \& Velandia, A. (2014). Ethnocentrism of the Colombian and Brazilian market and its relationship with the attitudes towards intention to purchase foreign and domestic products. Suma Psicológica, 21(2), 99-106. https://doi.org/10.1016/S0121-4381(14)70012-8

Nadiri, H., \& Tümer, M. (2010). Influence of ethnocentrism on consumers' intention to buy domestically produced goods: An empirical study in North Cyprus. Journal of Business Economics and Management, 11(3), 444-461. https://doi.org/10.3846/jbem.2010.22

Netemeyer, R.G., Durvasula, S., \& Lichtenstein, D.R. (1991). A Cross-National Assessment of the Reliability and Validity of the CETSCALE. Journal of Marketing Research, 28(3), 320-327. https://doi.org/10.2307/3172867

Olson, J., \& Jacoby, J. (1972). Cue utilization in the quality perception process. Proceedings of the Third Annual Conference of the of the Association for Consumer Research, 2(1), 23-31. https://doi.org/10.1108/eb026082

Ortega-Vivanco, M. (2020). Efectos del Covid-19 en el comportamiento del consumidor: Caso Ecuador. Retos Revista de Ciencias de la Administración y Economía, 10(20), pp. 233-247. https://doi.org/10.17163/ret.n20.2020.03 
Pham, H.C. (2020). Factors influencing the consumer buying behaviour in the competitive era: an empirical study of consumer durable in Vietnam. International Journal of Intelligent Enterprise, 8(1). https://doi.org/10.1504/IJIE.2021.112322

Rosero, C., \& Montalvo, H. (2015). La disonancia cognitiva como factor motivador en las decisiones del consumidor: caso smartphones. Retos Revista de Ciencias de Administración y Economía, 5(10), 177-192. https://doi.org/10.17163/ret.n10.2015.05

Shankarmahesh, M.N. (2006). Consumer ethnocentrism: An integrative review of its antecedents and consequences. International Marketing Review, 23(2), 146-172. https://doi.org/10.1108/02651330610660065

Sharma, S., Shimp, T.A., \& Shin, J. (1995). Consumer ethnocentrism: A test of antecedents and moderators. Journal of the Academy of Marketing Science, 23(1), 26-37. https://doi.org/10.1007/BF02894609

Shimp, T.A. (1984). Consumer Ethnocentrism: the Concept and a Preliminary Empirical test. Advances in Consumer Research, 11, 285-290.

Shimp, T.A., \& Sharma, S. (1987). Consumer Ethnocentrism: Construction and Validation of the CETSCALE. Journal of Marketing Research, 24(3), 280-289. https://doi.org/10.2307/3151638

Shoham, A., \& Makovec, M. (2003). Consumer Ethnocentrism, Attitudes, and Purchase Behavior. Journal of International Consumer Marketing, 15(4), 67-86. https://doi.org/10.1300/J046v15n04_04

Slovic, P., Fischhoff, B., \& Lichtenstein, S. (1977). Behavioral Decision Theory. Annual Review of Psychology, 28(1), 1-39. https://doi.org/10.1146/annurev.ps.28.020177.000245

Spillan, J.E., Kucukemiroglu, O., \& de Mayolo, C.A. (2007). Profiling peruvian consumers' lifestyles, market segmentation, and ethnocentrism. Latin American Business Review, 8(4), 38-59. https://doi.org/10.1080/10978520802114573

Sumner, W.G. (1906). Folkways. A study of the sociological importance of usages, manners, customs and morals. Psychological Bulletin, 13(1), 116-117. https://doi.org/10.1037/h0065334

Supphellen, M., \& Rittenburg, T. L. (2001). Consumer ethnocentrism when foreign products are better. Psychology and Marketing, 18(9), 907-927. https://doi.org/10.1002/mar.1035

Teng, W. (2019). Importance of corporate image for domestic brands moderated by consumer ethnocentrism.Journal of Product E Brand Management, 29(3) 257-272. https://doi.org/10.1108/JPBM-09-2018-2020

Wang, C.L., \& Chen, Z.X. (2004). Consumer ethnocentrism and willingness to buy domestic products in a developing country setting: Testing moderating effects. Journal of Consumer Marketing, 21(6), 391-400. https://doi.org/10.1108/07363760410558663

Wood, W., \& Neal, D.T. (2009). The habitual consumer. Journal of Consumer Psychology, 19(4), 579-592. https://doi.org/10.1016/j.jcps.2009.08.003

Wu, J., Zhu, N., \& Dai, Q. (2010). Consumer ethnocentrism, product attitudes and purchase intentions of domestic products in China... and Business Management, Chengdu, China ..., (Ebm), 2262-2265. https://bit.ly/2LYyKE7

Yagci, M.I. (2001). Evaluating the Effects of Country-of-Origin and Consumer Ethnocentrism. Journal of International Consumer Marketing, 13(3), 63-85. https://doi.org/10.1300/J046v13n03_05 